\title{
MicroRNA-21a-5p regulates TGF- $\beta 1$-induced myofibroblast transformation of rabbit keratocytes by targeting Smad7
}

\author{
Jianhai Bai $^{\mathrm{a}}$, Minli Zhang ${ }^{\mathrm{b}}$, Xiaomin Ding ${ }^{\mathrm{b}}$, Xingzhi Yang ${ }^{\mathrm{b}}$, Dan Zheng ${ }^{\mathrm{a}}$, Hongyun Mou $^{\mathrm{a}}$, Xiaoxiao Ruan ${ }^{\mathrm{a}}$, \\ Yueqing Feng ${ }^{\mathrm{c}}$, Haifei Han ${ }^{\mathrm{b}}$, Shili Wang ${ }^{\mathrm{a}, *}$ \\ a Department of Ophthalmology, Taizhou Central Hospital (Taizhou University Hospital), Zhejiang 318000 China \\ b Operating Room, Taizhou Central Hospital (Taizhou University Hospital), Zhejiang 318000 China \\ c Cardiothoracic Surgery, Taizhou Central Hospital (Taizhou University Hospital), Zhejiang 318000 China
}

*Corresponding author, e-mail: wangsl7149@tzzxyy.com

Received 29 Jan 2021

Accepted 27 Aug 2021

\begin{abstract}
The transforming growth factor $\beta 1$ (TGF- $\beta 1$ )-induced transformation of keratocytes to myofibroblasts is a very common process during corneal wound healing, but a dysregulated TGF- $\beta 1$ activity may induce unfavorable fibrosis and scar formation in the cornea. MicroRNA (miR)-21a-5p and Smad7 have been reported to play an important role in the fibrosis process. However, how miR-21a-5p involves in the TGF- $\beta 1$-induced myofibroblasts transformation of keratocytes remains unclear. In the present study, we found an increase in the expressions of $\alpha$-SMA and collagen I at protein level as well as Smad7 and miR-21a-5p at mRNA level when keratocytes were treated with TGF- $\beta 1$ Here; but the Smad7 at protein level kept unchanged. The overexpression of miR-21a-5p attenuated Smad7 protein expression, and miR-21a-5p inhibition promoted Smad7 protein expression without affecting its mRNA expression. Furthermore, inhibition of miR-21a-5p could decrease TGF- $\beta 1$-induced $\alpha$-SMA expression, whereas the addition of Smad7 knockdown would rescue the expression of $\alpha$-SMA. These results suggested that miR-21a-5p had a promoting effect on TGF- $\beta 1$ induced myofibroblast transformation of keratocytes by targeting Smad7 at its translation stage.
\end{abstract}

KEYWORDS: miR-21a-5p, keratocytes, TGF- $\beta 1$, corneal wound healing

\section{INTRODUCTION}

Corneal wound healing often occurs in response to various kinds of injuries and corneal transplantation, trauma, infection, or refractive surgery; and this dynamic complex process is strictly regulated by the interaction of keratocytes, stromal cells, extracellular matrix (ECM), cytokine, and growth factors [1]. It has been reported that TGF- $\beta 1$, the strongest-known pro-fibrotic molecule, involves in the regulation of ECM deposition and keratocytes activation, migration, and proliferation; while under pathological conditions, the secretion of TGF- $\beta 1$ is upregulated, which affects corneal wound healing process $[2,3]$. Keratocytes are in a resting state in the healthy cornea; but after an injury or surgery, they differentiate into myofibroblasts and have an ability to migrate to the wound site. However, an excessive number of myofibroblasts is undesirable because they may induce fibrotic scar formation. TGF- $\beta 1$ is one of the key factors in the transformation of keratocytes into myofibroblasts, and its regulatory function remains to be elucidated.

MicroRNA (miR or miRNA) is a class of small nonprotein-coding RNAs consisting of 20-23 nucleotides and acting as negative regulators of gene expression by directly binding to the $3^{\prime}$ UTR of the target mRNA and then causing degradation or translational inhibition of such particular mRNA [4]. The regulatory role of miR in TGF- $\beta$-related wound healing has been reported. For example, Chen et al found that lowexpression of miR-203a in early-gestation benefited cutaneous scarless wound healing by regulating TGF- $\beta$ signaling [5]. In addition, miR-21a-5p is well-known as the facilitator of fibrosis development via altered expression of genes involved in biological and pathological processes of cells such as growth, proliferation, differentiation, apoptosis, and stress resistance, and it has been found to promote the activation of Smads signaling and spinal fibrosis after mechanical trauma [6-8]. A predicated miR-21a-5p target site is in the $3^{\prime}$ UTR of Smad7. Consequently, as an inhibitor of TGF$\beta 1$ signaling, Samd7 has a negative effect on TGF- $\beta 1$ induced processes.

In this study, we investigated whether miR$21 \mathrm{a}-5 \mathrm{p}$ involves in TGF- $\beta 1$-induced keratocyte-tomyofibroblast transition by targeting Smad7. Keratocytes were treated with TGF- $\beta 1$, and then the expressions of $\alpha$-SMA and collagen I were detected to confirm the myofibroblast differentiation. After that, the effects of TGF- $\beta 1$ and miR-21a-5p on Smad7 expression at protein and mRNA levels were assessed, respectively. Then, the extent of TGF- $\beta 1$-induced transformation of keratocytes to myofibroblasts was investigated under the simultaneous action of miR-21a-5p inhibition and Smad7 knockdown.

\section{MATERIALS AND METHODS}

Reagents

Dulbecco's Modified Eagle's Medium (DMEM) with high glucose and fetal bovine serum (FBS) were purchased from Gibco Company (Grand Island, NY, USA). 
Recombinant human TGF- $\beta 1$ protein and anti- $\alpha$-SMA, anti-collagen I, anti-Smad7, anti-GAPDH primary antibodies were obtained from Abcam Company (Cambridge, USA). HRP-labeled secondary antibody and cell counting kit-8 (CCK-8) were purchased from Beyotime Institute of Biotechnology (Haimen, China). The syntheses of miR-21 mimic, miR-21 inhibitor, negative control (NC), Smad7 siRNA, and NC-siRNA were completed by RiboBio Company (Guangzhou, China). Other commonly used reagents were obtained from commercial sources without further purification.

\section{Cell culture and transfection}

Keratocytes were isolated from the corneas excised from rabbit eyes according to the previously reported method [9]. Keratocytes were cultured in DMEM containing $10 \%$ FBS and kept in a humidified 5\% $\mathrm{CO}_{2}$ incubator at $37^{\circ} \mathrm{C}$, and the medium was changed a few times every week. Cells in logarithmic phase were chosen for further experiments. Keratocytes were transfected with miR-21 mimic (40 nM), inhibitor $(60 \mathrm{nM})$, and NC (60 nM) using Lipofectamine 2000 following the manufacturer's instructions [10]. Smad7 siRNA and NC-siRNA were individually used, or in combination with miR-21 inhibitor in the transient transfection, and the efficacy of Smad7 knockdown with siRNA was confirmed by Western blot assay.

\section{Cell grouping and TGF- $\beta 1$ treatment}

For TGF- $\beta 1$ induced myofibroblast transformation of rabbit keratocytes, the keratocytes were washed twice with PBS and then divided into three groups: the PBS treated control group, and the other two groups subjected to $2 \mathrm{ng} / \mathrm{ml}$ of TGF- $\beta 1$ for $24 \mathrm{~h}$ and $48 \mathrm{~h}$, respectively. After treatment, the expressions of $\alpha$ SMA and collagen I, two commonly used molecular markers of myofibroblast, in the three groups were measured using Western blot assay.

To observe the effects of TGF- $\beta 1$ on Smad7 and miR-21a-5p expressions, keratocytes were cultured in medium containing $2 \mathrm{ng} / \mathrm{ml}$ of TGF- $\beta 1$ for $48 \mathrm{~h}$, and then miR-21a-5p and Smad7 mRNA were identified by RT-PCR. Smad7 protein was measured using Western blot assay.

To study the effects of miR-21a-5p on the expression of Smad7, all the three keratocyte groups were transfected with miR-21 mimic, inhibitor, or NC; and then Smad7 expressions at mRNA level and protein level were investigated by RT-PCR and Western blot assay, respectively.

The roles of miR-21a-5p and Smad7 in the TGF$\beta 1$-induced transformation of keratocytes to myofibroblasts were investigated. Keratocytes were divided into four groups: the control and the other three respectively treated with $2 \mathrm{ng} / \mathrm{ml}$ of TGF- $\beta 1$, miR21 inhibitor, and Smad7 knockdown. The cells were cultured for $48 \mathrm{~h}$, and then the $\alpha$-SMA mRNA and its protein were detected.

\section{Western blot assay}

After receiving corresponding treatments, keratocytes in individual groups were harvested and subjected to Western blot analysis as described in our previously reported procedures [11].

\section{RNA isolation and quantitative RT-PCR}

To assess the miR and the mRNA expressions, total RNA was first isolated from the keratocytes, and then its concentration and quality were measured using the ultraviolet spectrophotometry. The expression of miR-21a-5p was detected using the quantitative real-time PCR (qRT-PCR) following the instructions of miRNA Reverse Transcription Kit. For the mRNA detection, mRNAs of Smad7, $\alpha$-SMA, and GAPDH were reverse-transcribed using the PrimeScript RT Reagent Kit, and then the expression levels were measured by qRT-PCR using a 7500 RTPCR system. The primer pairs using the reported sequences were as follows: miR-21a-5p, forward: 5'-ACACTCCAGCTGGGTAGCTTATCAGACTGA-3', reverse: $5^{\prime}$-CTCAACTGGTGTCGTGGAGTCGGCAATTC AGTTGAGGATTATGA-3'; Smad7, forward: $5^{\prime}$-GTG GCATACTGGAGGAGAA-3', reverse: $5^{\prime}$-GATGGAG AACCAGGGAACA-3 ${ }^{\prime} ; \quad \alpha$-SMA, forward: $5^{\prime}$-TTCA ATGTCCCAGCCATGTA-3', reverse: $5^{\prime}$-TGCCAGTT GTACGTCCAGAG-3'; and GAPDH, forward: $5^{\prime}$-GC ACCGTCAGGCTGAGAAC-3' , reverse: $5^{\prime}$-ATGGTG GTAAGACGCCAGT- $3^{\prime}$ ). Threshold cycle (Ct) data were collected, and the fold of target mRNA relative to GAPDH mRNA was measured using the $2^{-\Delta \Delta \mathrm{Ct}}$.

\section{Cell proliferation assay}

Keratocytes transfected with miR-21 mimic, miR-21 inhibitor or NC were seeded in 96-well plate at a density of 3000 cells/well $(100 \mu \mathrm{l})$, and then cultured for 24,48 and $72 \mathrm{~h}$ in a standard culture condition. At each time point, $10 \mu \mathrm{l}$ of CCK- 8 solution was added into each well, and the absorbance at $450 \mathrm{~nm}$ was measured at $2 \mathrm{~h}$ after the addition using a micro-plate reader. The absorbance value represents the relative proliferation ability of cells. All experiments were repeated in tripicates.

\section{Statistical analysis}

All data were expressed as mean $\pm S D$, and the differences between different groups were analyzed using unpaired Student's $t$-test (two groups) or one-way analysis of variance (ANOVA) followed with LSD- $t$ post-hoc test (more than two groups). When $p$ value was less than 0.05 , the difference was considered statistically significant. 


\section{RESULTS AND DISCUSSION}

\section{TGF- $\beta 1$ induced myofibroblast transformation of rabbit keratocytes}

TGF- $\beta 1$ signaling is a critical driver in mediating collagen accumulation, which is important for the wound repair in many organs; but an out-of-control TGF$\beta 1$ activity causes undesirable tissue fibrosis, such as pulmonary fibrosis [12]. Besides, the fibrotic response induced by corneal wound healing process will compromise visual performance, of which the transitional activated fibroblast of TGF- $\beta 1$ induced phenotype transformation of corneal keratocytes to myofibroblast plays an important role [13]. The excessively up-regualted TGF- $\beta 1$ signaling leads to fibrotic wound healing and induction of scar formation. Therefore, TGF- $\beta 1$ is often chosen to establish the activation of myofibroblasts transformation model of keratocytes. The keratocytes derived myofibroblasts not only have obvious phenotypic changes, but also express some intermediate filament proteins, e.g. $\alpha$-SMA, vimentin, and desmin; and excessive ECM, such as collagen I and fibronectin. In fact, $\alpha$-SMA and collagen I are the two most commonly used markers for myofibroblasts identification.

In present study, the effects of TGF- $\beta 1$ stimulation on $\alpha$-SMA and collagen I expressions were investigated to assess the cell phenotype of keratocytes. As shown in Fig. 1, the expressions of $\alpha$-SMA and collagen I were up-regulated in a time-dependent manner when rabbit keratocytes were treated with $2 \mathrm{ng} / \mathrm{ml}$ of TGF$\beta 1$, suggesting TGF- $\beta 1$-induced keratocyte activation. In addition to the increase of $\alpha$-SMA and collagen I expressions, it has been reported that corneal keratocytes treated with TGF- $\beta 1$ also showed other fibrosis-related changes including ECM stiffness [14].

TGF- $\beta 1$ promotes miR-21a-5p and Smad7 mRNA expression, but has no effect on the expression of Smad7 protein

The fibrosis-related miR-21a-5p has been reported to be involved in the regulation of fibrosis in multiple tissues, such as spine, uterus, lung $[6,7,15]$. TGF$\beta /$ Smad signaling is widely recognized as a core pathway in the process of fibrosis-associated cell activation and plays a pivotal role in the onset and development of fibrosis. Smad7 is a member of the Smad family, and also a major inhibitory regulator of TGF$\beta$ signaling. Previous studies showed that miR-21a$5 \mathrm{p}$, as an upstream regulatory miR had a promotional effect on TGF- $\beta 1$ signaling by targeting inhibition of Smad7. Interestingly, Smad7, mostly located in the nucleus, can be translocated to the cytoplasm under the stimulation of TGF- $\beta 1$ [16]. After the TGF- $\beta 1$ induced keratocytes activation was confirmed, we further investigated the expressions of miR-21a-5p and Smad7 and their roles at different levels. We found that when keratocytes were treated with TGF- $\beta 1$ for
$48 \mathrm{~h}$, the protein expression of Smad7 remained stable (Figs. 2A and 2B), but both miR-21a-5p and Smad7 mRNA increased significantly (Figs. $2 \mathrm{C}$ and 2D). The results suggested that TGF- $\beta 1$ stimulated the expressions of miR-21a-5p and the Smad7 mRNA in keratocytes, but surprisingly, the expression of Smad7 at protein level did not increase. The inconsistency of mRNA and protein expressions indicated that Smad7 was probably regulated negatively at mRNA level.

\section{Smad7 is the target of miR-21a-5p}

Smad7 has been considered as a target of miR-21a$5 p$ in different fibrosis processes $[17,18]$. To explore whether miR-21a-5p also had a regulatory effect on Smad7 in the TGF- $\beta 1$-induced myofibroblasts transformation of keratocytes, cells were transfected with miR-21a-5p mimic, miR-21a-5p inhibitor or NC, and then Smad7 expression was detected. Fig. 3A shows that miR-21a-5p had little to negligible influence on the proliferation of keratocytes. Smad7 mRNA in keratocytes transfected with miR-21a-5p mimic or inhibitor had no significant change (Fig. 3B). However, the expression of Smad7 at protein level in miR-21a-5p mimic group was inhibited, but enhanced in miR-21a$5 p$ inhibitor group (Figs. 3C and 3D). These results demonstrated that Smad7 is a direct target of miR-21a$5 p$ in the keratocyte transformation, and miR-21a-5p may regulate Smad7 protein expression via producing a negative effect on its protein translation.

miR-21a-5p involves in the TGF- $\beta 1$-induced myofibroblast transformation of keratocytes by targeting Smad7

Since miR-21a-5p is a negative regulator of Smad7 mRNA, the role of miR-21a-5p/Smad7 in TGF- $\beta 1$ induced keratocytes activation is worth studying. The efficiency of Smad7 knockdown was confirmed by Western blot assay (Fig. 4A). The fibrotic marker $\alpha$ SMA was chosen to reflect the activation level of keratocytes. As shown in Figs. 4B and 4C, miR-21a-5p inhibitor can inhibit the enhanced $\alpha$-SMA expression at protein and mRNA levels, which were induced by TGF- $\beta 1$ treatment. But when Smad7 was knocked down with siRNA, the inhibitory effect was partially relieved. These results supported the opinion that miR-21a-5p involved in the process of myofibroblasts transformation of keratocytes by targeting Smad7.

The activation or myofibroblasts transformation of keratocytes is an essential part of corneal wound healing, and exploring its underlying molecular mechanisms will contribute to understandings of the process and future development of new therapeutic strategies. Smad7 is a key regulator of TGF- $\beta$-induced fibrogenesis, and Smad7 overexpression can accelerate corneal endothelial wound closure. However, over inhibition of Smad7 can cause an undesirable, pathogenic response of wound healing in the cornea [19]. Our 

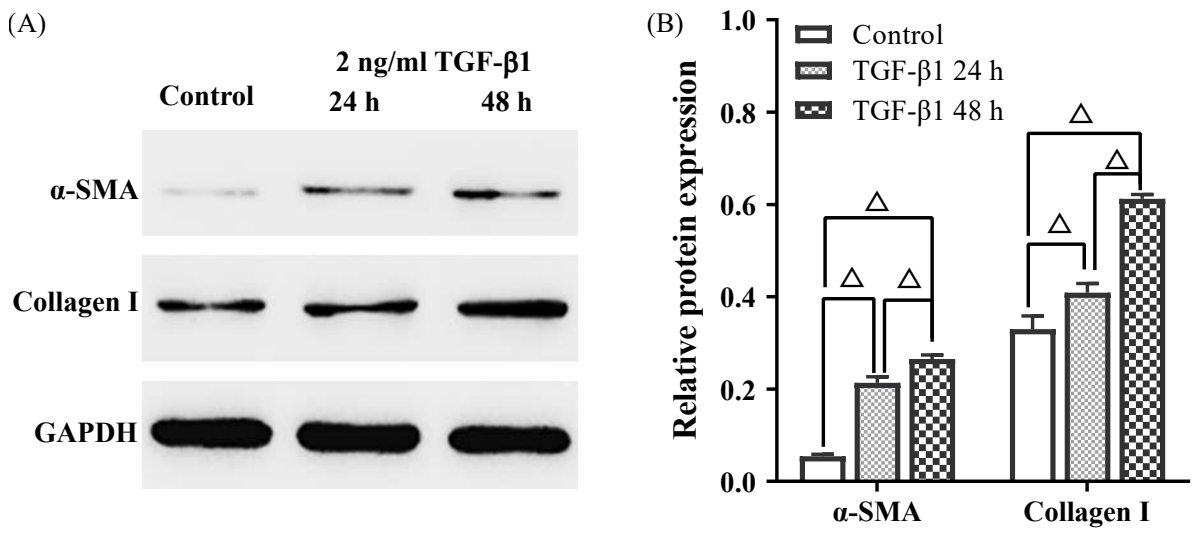

Fig. 1 TGF- $\beta 1$ induced myofibroblast transformation. Two commonly used molecular markers of myofibroblast, $\alpha$-SMA and collagen I in keratocytes treated without or with TGF- $\beta 1$ for 24 and 48 h were detected using Western blot assay. $n=3$, $\Delta p<0.05$.
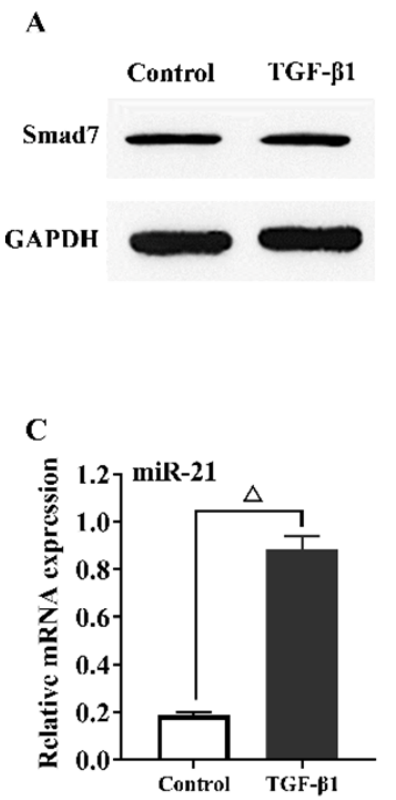

B

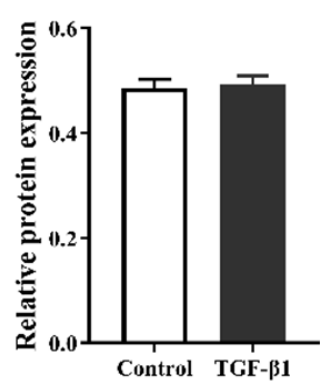

D

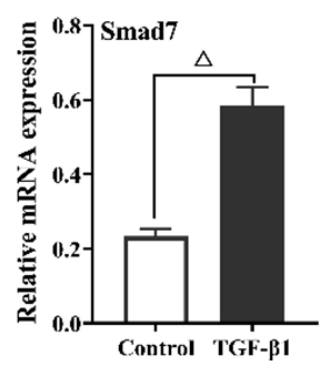

Fig. 2 Effects of TGF- $\beta 1$ on Smad7 and miR-21a-5p expressions in keratocytes. TGF- $\beta 1$ did not affect Smad7 protein expression (A,B), but increase miR-21a-5p (C) and Smad7 (D) expressions at mRNA level. $n=3, \Delta p<0.05$.

present study confirmed that without the negative feedback regulation of Smad7, TGF- $\beta 1$-induced fibrosis response would lose control and inhibition of miR21a-5p also cannot slow down this deleterious process. The cross-talk between TGF- $\beta 1 /$ Smad7 and miR-21a$5 p$ emphasized the importance of miR-21a-5p in regulation of the transition from keratocytes to myofibroblasts. Therefore, miR-21a-5p may become an effective therapeutics target for the fibrosis formation in cornea wound healing.

$\mathbf{A}$

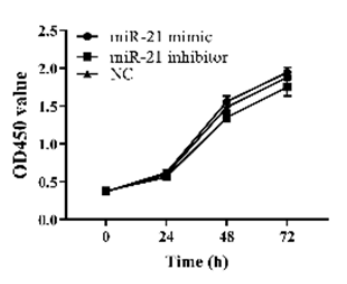

B

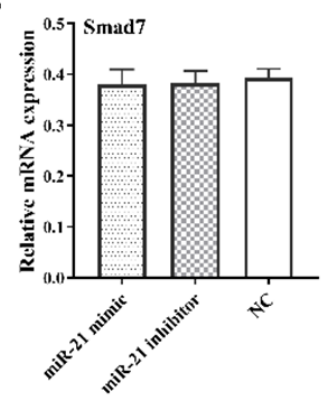

C
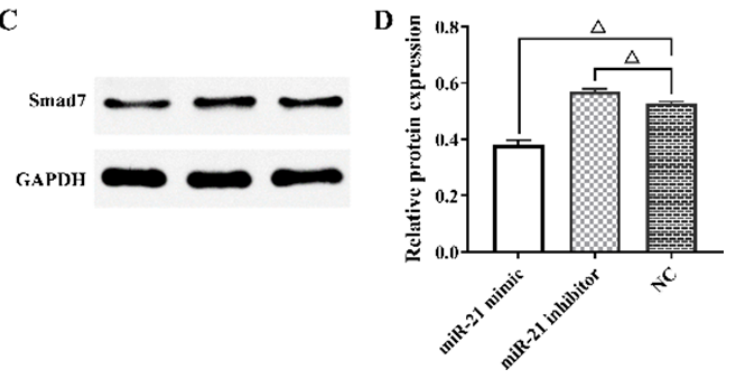

Fig. 3 MiR-21a-5p regulates Smad7 protein expression by inhibiting its translation. The proliferation rate (A), Smad7 expression at mRNA level (B), and protein level (C,D) of keratocytes infected with miR-21a-5p mimic, miR-21a-5p inhibitor, or NC. $n=3, \Delta p<0.05$.

\section{CONCLUSION}

In conclusion, our present study showed that miR-21a$5 p$ plays a positive regulator role in TGF- $\beta 1$-induced myofibroblasts transformation of keratocytes by targeting Smad7, suggesting that miR-21a-5p involves in the signaling pathway associated with stromal wound healing process, and the inhibition of miR-21a-5p may relieve the fibrotic scar formation. 

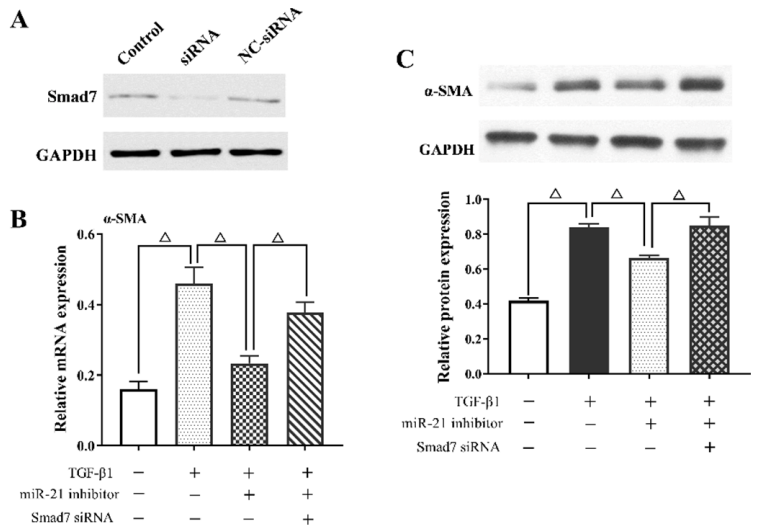

Fig. 4 Smad7 knockdown rescued TGF- $\beta 1$-induced myofibroblast transformation repressed by miR-21a-5p inhibitor. Representative Western blot of Smad7 knockdown in keratocytes (A), and the expression of $\alpha$-SMA at mRNA level (B) and protein level (C) in keratocytes transfected with miR-21a-5p inhibitor without or with Smad7 knockdown after treatment with TGF- $\beta 1 . n=3, \Delta p<0.05$.

Acknowledgements: This study was financially supported by Taizhou Science and Technology Bureau Project (Grant No. 1901ky37).

\section{REFERENCES}

1. Wilson SE, Mohan RR, Mohan RR, Ambrosio R Jr, Hong J, Lee J (2001) The corneal wound healing response: cytokine-mediated interaction of the epithelium, stroma, and inflammatory cells. Prog Retin Eye Res 20, 625-637.

2. Jeon KI, Hindman HB, Bubel T, McDaniel T, DeMagistris M, Callan C, Huxlin KR (2018) Corneal myofibroblasts inhibit regenerating nerves during wound healing. Sci Rep 8, ID 12945.

3. Xing D, Bonanno JA (2009) Hypoxia reduces TGFbeta1induced corneal keratocyte myofibroblast transformation. Mol Vis 15, 1827-1834.

4. O’Brien J, Hayder H, Zayed Y, Peng C (2018) Overview of microRNA biogenesis, mechanisms of actions, and circulation. Front Endocrinol (Lausanne) 9, ID 402.

5. Chen M, Zhao F, Wang Z, Lang H, Zhang K, Zhang T, Wang R, Shi P, et al (2020) Low-expression of microRNA203a in mid-gestational human fetal keratinocytes contributes to cutaneous scarless wound healing by targeting Tenascin-C. ScienceAsia 46, 133-141.

6. Wang W, Tang S, Li H, Liu R, Su Y, Shen L, Sun M, Ning B (2018) MicroRNA-21a-5p promotes fibrosis in spinal fibroblasts after mechanical trauma. Exp Cell Res 370, 24-30.
7. Cardozo ER, Foster R, Karmon AE, Lee AE, Gatune LW, Rueda BR, Styer AK (2018) MicroRNA 21a-5p overexpression impacts mediators of extracellular matrix formation in uterine leiomyoma. Reprod Biol Endocrinol 16, ID 46.

8. Schauerte C, Hubner A, Rong S, Wang S, Shushakova N, Mengel M, Dettling A, Bang C, et al (2017) Antagonism of profibrotic microRNA-21 improves outcome of murine chronic renal allograft dysfunction. Kidney Int 92, 646-656.

9. Ge H, Yin X, Fu A, Zhang H, Song Z, Li Z, Liu P (2007) Improving ways to cryopreservation and resuscitation of keratocytes of rabbit in vitro. Recent Adv Ophthalmol 27, 670-674.

10. Liu L, Qian H (2015) Up-regulation of miR-21 promotes cell proliferation and collagen synthesis in pulmonary fibroblasts. Chin J Cell Mol Immunol 31, 918-922.

11. Bai J, Xu Y, Dieo Y, Sun G (2019) Combined low-dose $\mathrm{LiCl}$ and LY294002 for the treatment of osteoporosis in ovariectomized rats. J Orthop Surg Res 14, ID 177.

12. Lin S, Fu J, Wang C, Liu Q, Xu F, Yang Y (2020) Inhibition of transforming growth factor- $\beta$ type I receptor signaling by RNA interference suppresses the proliferation, collagen synthesis, and inflammation factors in human embryonic lung fibroblasts. ScienceAsia 46, 472-480.

13. Jester JV, Huang J, Petroll WM, Cavanagh HD (2002) TGF $\beta$ induced myofibroblast differentiation of rabbit keratocytes requires synergistic TGF $\beta$, PDGF and integrin signaling. Exp Eye Res 75, 645-657.

14. Maruri DP, Miron-Mendoza M, Kivanany PB, Hack JM, Schmidtke DW, Petroll WM, Varner VD (2020) ECM Stiffness controls the activation and contractility of corneal keratocytes in response to TGF- $\beta 1$. Biophys $J$ 119, 1865-1877.

15. Makiguchi T, Yamada M, Yoshioka Y, Sugiura H, Ichinose M (2016) Serum extracellular vesicular miR-21-5p is a predictor of the prognosis in idiopathic pulmonary fibrosis. Resp Res 17, ID 110.

16. Landstrom M, Heldin NE, Bu S, Hermansson A, Itoh S, ten Dijke P, Heldin CH (2000) Smad7 mediates apoptosis induced by transforming growth factor beta in prostatic carcinoma cells. Curr Biol 10, 535-538.

17. Cui H, He Y, Chen S, Zhang D, Yu Y, Fan C (2019) Macrophage-derived miRNA-containing exosomes induce peritendinous fibrosis after tendon injury through the miR-21-5p/Smad7 pathway. Mol Ther Nucleic Acids 14, 114-130.

18. Wang W, Liu R, Su Y, Li H, Xie W, Ning B (2018) MicroRNA-21-5p mediates TGF-beta-regulated fibrogenic activation of spinal fibroblasts and the formation of fibrotic scars after spinal cord injury. Int J Biol Sci 14, 178-188.

19. Funaki T, Nakao A, Ebihara N, Setoguchi Y, Fukuchi Y, Okumura K, Ra C, Ogawa H, et al (2003) Smad7 suppresses the inhibitory effect of TGF-beta2 on corneal endothelial cell proliferation and accelerates corneal endothelial wound closure in vitro. Cornea 22, 153-159. 